\title{
Measurement of a broadband input coupler for a W-band gyro-TWA
}

\author{
Liang Zhang, Craig R. Donaldson, and \\ Adrian W. Cross \\ Department of Physics, SUPA, \\ University of Strathclyde \\ Glasgow, UK \\ liang.zhang@strath.ac.uk \\ craig.donaldson@strath.ac.uk \\ a.w.cross@strath.ac.uk
}

\author{
Wenlong $\mathrm{He}$ \\ College of Electronic Science and \\ Technology \\ Shenzhen University \\ Shenzhen, China \\ wenlong.he@szu.edu.cn
}

\begin{abstract}
In this paper, the design and measurement results of the input coupling system for a W-band gyro-TWA is represented. The coupling system was designed to achieve $10 \%$ bandwidth centered at $95 \mathrm{GHz}$. In the measurement, an average transmission coefficient of $\mathbf{- 2 . 0} \mathrm{dB}$ was measured over the designed frequency range.
\end{abstract}

Keywords-gyrotron traveling wave amplifier, input coupler, helically corrugated waveguide, broadband amplification

\section{INTRODUCTION}

A Gyrotron traveling wave amplifier (gyro-TWA) is capable to generate high-power millimeter wave radiation with bandwidth. It can be used in applications such as telecommunication, RADAR, plasma diagnostics, and electron paramagnetic resonance spectroscopy. At the University Strathclyde, a W-band gyro-TWA using a helically corrugated interaction region (HCIR) has been developed [1]. Due to the unique property of the HCIR that is able to couple $\mathrm{TE}_{11}$ and $\mathrm{TE}_{21}$ modes to generate an eigenmode that has nearly constant group velocity over a large bandwidth, the gyro-TWA is able to operate over $10 \%$ bandwidth.

Fig. 1 shows the schematic drawing of the W-band gyroTWA. It contains a cusp electron gun as well two coils with opposite magnetic fields to generate a large orbit electron beam. The axial encircling large orbit electron beam allows a $2^{\text {nd }}$ cyclotron harmonic beam-wave interaction, therefore, it is able to reduce the requirement of the magnetic field strength $[2,3]$.

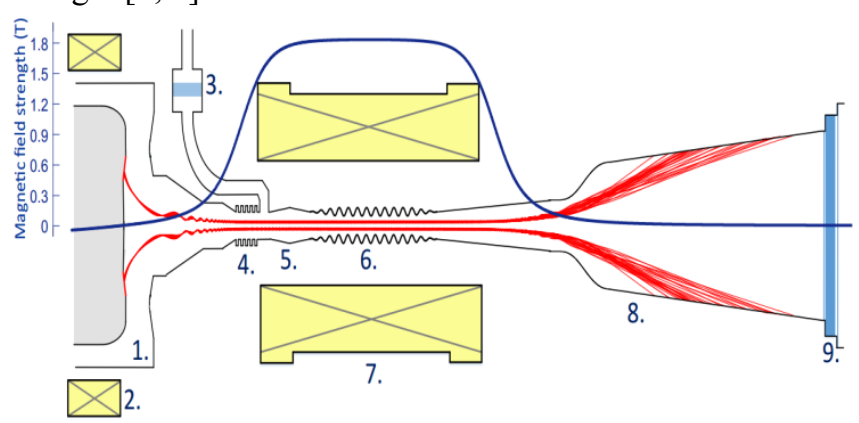

Fig. 1. The schematic of the W-band gyro-TWA. Its main components include (1) cusp electron gun, (2) reverse coil, (3) pillbox-type microwave window, (4) broadband reflector, (5) elliptical polarizer, (6) helically corrugated interaction region, (7) main coil, (8) smooth-profiled horn, (9) multiple-layer microwave window.

The input seed microwave signal was generated by a solid-state source and then fed into the gyro-TWA system through a pillbox type microwave window [4], a waveguide bend as well as a T-junction side-wall coupler [5]. An elliptical polarizer was used to convert the linearly polarized $\mathrm{TE}_{11}$ wave into a circularly polarized one for the beam-wave interaction. The input microwave signal was amplified in the HCIR, and the high power radiation is converted into the fundamental Gaussian mode by using a smooth-profiled horn and coupled out from a wide-band multiple-layer microwave window $[6,7]$.

The broadband amplification of the gyro-TWA requires that all the components have wide bandwidth performance. All the components require to have minimum reflection to avoid possible oscillation with more than $10 \%$ frequency bandwidth centered at $95 \mathrm{GHz}$. Before the components were assembled or brazed together, their performance were measured individually by a vector network analyzer (VNA). The components included a pillbox window, a waveguide bend, two elliptical polarizers, an HCIR and a waveguide taper.

Fig. 2 shows the pillbox window after brazing. The pillbox window was designed as demountable therefore can be reused in other experiments. A custom-sized stainless flange with CF-like knife edge was vacuum brazed onto the rectangular waveguide to seal the vacuum. On the other side, a standard WR-10 UG-387/U round flange was used to connect with the other components, for example, the VNA port or the solid state source. In the measurement, the reflection was about $-15 \mathrm{~dB}$ and the transmission loss was about $-0.5 \mathrm{~dB}$. A summary of the loss from the individual component is listed in Table 1.

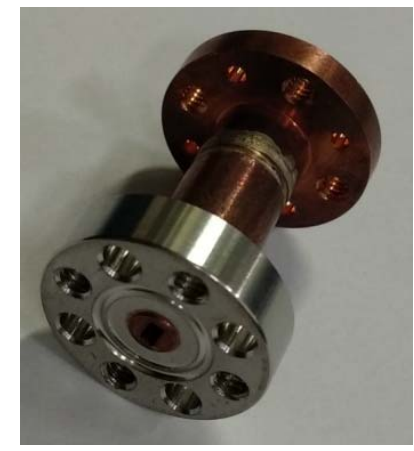

Fig. 2. The pillbox window after brazing.

Fig. 3 shows the measurement setup of the input coupling system, and the measured transmission and reflection results are shown in Fig. 4. The transmission coefficient of the whole measuring circuit is better than $-6 \mathrm{~dB}$ over the interested frequency of $90-100 \mathrm{GHz}$, which agreed with the sum of the loss of individual components. The loss of the whole input coupling system is about $-2.0 \mathrm{~dB}$, which 
includes the loss from the pillbox window and the waveguide bends, but exclude the loss of the helically corrugated waveguide and the elliptical polarizers.

TABLE I. LOSS OF INDIVIDUAL COMPOMENT

\begin{tabular}{|l|l|}
\hline Component & Loss (dB) \\
\hline pillbox window & 0.6 \\
\hline waveguide bend & 0.4 \\
\hline elliptical polarizers 1 & 0.9 \\
\hline HCIR & 1.5 \\
\hline waveguide taper & 1.0 \\
\hline elliptical polarizers 2 & 0.6 \\
\hline circular-to-rectangular converter & 0.3 \\
\hline
\end{tabular}

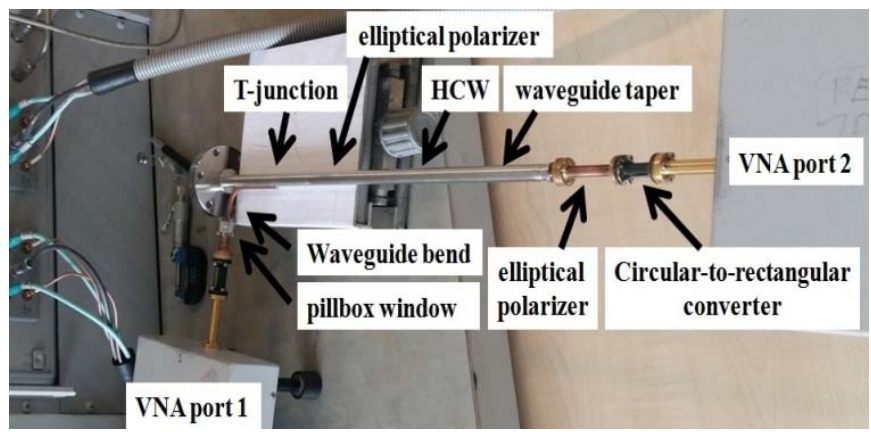

Fig. 3. The experimental setup of the input coupling system.

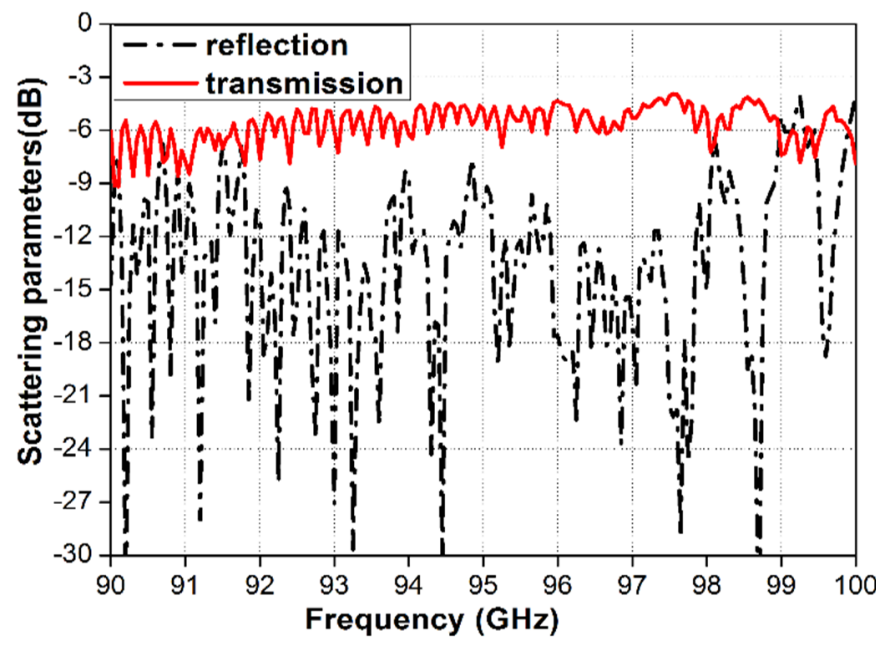

Fig. 4. The scattering parameters of the measurement.

The input coupling system was then used in the gyroTWA experiment and achieve broadband amplification as predicted. When the gyro-TWA was driven by a $55 \mathrm{kV}, 1.5$ A electron beam, it achieved a $3-\mathrm{dB}$ gain bandwidth of more than $5.5 \mathrm{GHz}$ in the measurement due to the limitation of the solid-state source. A further experiment demonstrating the frequency agility of the gyro-TWA showing good agreement of the phase of the input frequency-swept signal and output radiation is summarized in reference [8].

\section{CONCLUSION}

In this paper, a broadband input coupling system was manufactured and measured for a W-band gyro-TWA. After brazing and assembling, the input coupling system was leak tested with vacuum lower than $1 \mathrm{E}-9$ mbar measured. The millimeter wave properties of the components were measured using a vector network analyzer and the results agreed well with the simulation prediction. The overall loss of the input coupling system was found to be about $-2 \mathrm{~dB}$.

\section{ACKNOWLEDGMENT}

This work was supported by EPSRC UK (research Grant No. EP/G036659/1, EP/K029746/1) and STFC UK (research Grant No. ST/N002326/1 and ST/P001890/1).

\section{REFERENCES}

[1] W. He, C. R. Donaldson, L. Zhang, K. Ronald, A. D. R. Phelps, and A. W. Cross, "Broadband Amplification of Low-Terahertz Signals Using Axis-Encircling Electrons in a Helically Corrugated Interaction Region," Phys. Rev. Lett., vol. 119, no. 18, p. 184801, Oct. 2017. DOI: 10.1103/PhysRevLett.119.184801.

[2] C. R. Donaldson, W. He, A. W. Cross, F. Li, A. D. R. Phelps, L. Zhang, K. Ronald, C. W. Robertson, C. G. Whyte, and A. R. Young, "A cusp electron gun for millimeter wave gyrodevices," Appl. Phys. Lett., vol. 96, no. 14, p. 141501, 2010. DOI: 10.1063/1.3374888.

[3] L. Zhang, W. He, C. R. Donaldson, and A. W. Cross, "Investigation on the optimal magnetic field of a cusp electron gun for a W-band gyro-TWA," Physics of Plasmas, vol. 25, no. 5, p. 053104, 2018/05/01 2018. DOI: 10.1063/1.5027070.

[4] L. Zhang, C. R. Donaldson, A. W. Cross, and W. He, "A pillbox window with impedance matching sections for a W-band gyroTWA," IEEE Electron Device Letters, pp. 1-1, 2018. DOI: 10.1109/LED.2018.2834859.

[5] L. Zhang, W. He, C. R. Donaldson, J. R. Garner, P. McElhinney, and A. W. Cross, "Design and Measurement of a Broadband Sidewall Coupler for a W-Band Gyro-TWA," IEEE Trans. Microwave Theory Techn., vol. 63, no. 10, pp. 3183-3190, Oct. 2015. DOI: 10.1109/TMTT.2015.2464302.

[6] L. Zhang, W. He, C. R. Donaldson, G. M. Smith, D. A. Robertson, R. I. Hunter, and A. W. Cross, "Optimization and Measurement of a Smoothly Profiled Horn for a W-Band Gyro-TWA," IEEE Trans. Electron Devices, vol. 64, no. 6, pp. 2665-2669, June 2017. DOI: 10.1109/TED.2017.2687949.

[7] C. R. Donaldson, P. McElhinney, L. Zhang, and W. He, "Wide-Band HE11 Mode Terahertz Wave Windows for Gyro-Amplifiers," IEEE Transactions on Terahertz Science and Technology, vol. 6, no. 1, pp. 108-112, June 2016. DOI: 10.1109/TTHZ.2015.2495221.

[8] L. Zhang, C. R. Donaldson, P. Cain, A. W. Cross, and W. He, "Amplification of frequency-swept signals in a W-band gyrotron travelling wave amplifier," IEEE Electron Device Letters, pp. 1-1, 2018. DOI: 10.1109/LED.2018.2836868. 